\title{
Eficacia de la férula nocturna y el ultrasonido para tratar el síndrome del túnel carpiano. Estudio clínico controlado y aleatorizado
}

\author{
Daniel Hernandez, Esteban Terpolilli, Daniel H. Verdecchia, Valeria Leiva, Laura Ventos, Romina Rodríguez, \\ Anabella Gamboa, María Belén Spath, Jimena Steimberg, Martín Sanz, Erica Williams, Sabrina Mohr, Paola Colesnik, \\ Tai Lo Chen, Silvina Dell'Era, Mariana Dimaro, Romina Díaz, Bruno Baldo, Matías Accoce \\ Unidad de Kinesiología, Hospital General de Agudos "Dr. Carlos G. Durand”, Ciudad Autónoma de Buenos Aires, Argentina
}

\begin{abstract}
RESUMEN
Objetivo: Comparar la eficacia de la inmovilización nocturna de la muñeca con una férula cubital en ángulo neutro junto con la aplicación de ultrasonido en pacientes con síndrome del túnel carpiano leve y moderado. Materiales y Métodos: Entre octubre de 2007 y marzo de 2010, se incluyó a pacientes > 18 años con síndrome del túnel carpiano confirmado por electromiografía en un hospital de Buenos Aires. Se realizó una aleatorización estratificada, con bloques permutados aleatorios, y apareamiento por sexo y edad. Los pacientes fueron asignados al grupo experimental (GE) o al grupo de control (GC). Ambos grupos recibieron ultrasonido de $1 \mathrm{MHz}$ pulsante por $15 \mathrm{~min}$, 3 veces por semana, durante 6 semanas. Los pacientes del GE, además, utilizaron una férula nocturna. Se evaluaron el dolor y la parestesia con la escala analógica visual de $100 \mathrm{~mm}$, la PSFS y el test de Moberg, al comenzar, a las 3 semanas y, al finalizar, a las 6 semanas, y durante el seguimiento, al mes, y a los 3 y 6 meses, con evaluador a ciego. Resultados: Se analizó a 32 pacientes del GC y a 33 del GE. Al finalizar el tratamiento, todas las variables habían mejorado en ambos grupos, con diferencia de medias estadísticamente significativa para el dolor a favor del GE a las 3 semanas de tratamiento 1,64 (IC95\% 0,38-2,91; p = 0,012), pero sin diferencia clínica significativa. No se informaron efectos adversos. Conclusión: El tratamiento con una férula nocturna y ultrasonido no es superior al ultrasonido solo en pacientes con STC.
\end{abstract}

Palabras clave: Síndrome del túnel carpiano; tratamiento; férulas; terapia con ultrasonido.

Nivel de Evidencia: II

Night splinting effectiveness in Carpal Tunnel Syndrome. A randomized controlled clinical trial

\begin{abstract}
Objective: To compare the effectiveness of night wrist immobilization using an ulnar splint in neutral angle versus the use of ultrasound (US) in patients with Carpal Tunnel Syndrome (CTS). Materials and Methods: Study population included over 18 years of age that were treated for electromyography-confirmed CTS between October 2007 and March 2010 at a Buenos Aires hospital. A sex- and age-stratified randomization was performed by using randomly permuted blocks, allocating patients into the experimental group (EG) and control group (CG). Pulsed US therapy was administered for 15 minutes to all patients three times a week for six weeks at a frequency of $1 \mathrm{MHz}$. In addition, EG patients were also prescribed night splint. Pain and paresthesia were evaluated using a 100mm Visual Analogue Scale (VAS), the Patient Specific Functional Scale (PSFS), and the Moberg pickup test (MPUT) at baseline, at 3 and 6 weeks, and at 3 and 6 months after treatment institution by a blinded investigator. Results: Study population consisted of 85 cases ( 65 patients) that were randomly allocated to $C G(n=42)$ or $E G(n=43)$. Improvement of all the variables was observed at the end of treatment in both groups, with a $1.64(95 \% \mathrm{Cl}: 0.38-2.91, \mathrm{P}=0.012)$ statistically significant difference in means for pain in favor of the EG at 3 weeks of treatment, but without a significant clinical difference. No adverse effects were observed. Conclusion: The effectiveness of combined night splint and US therapy is not superior to the US alone treatment in CTS patients.
\end{abstract}

Key words: Carpal tunnel syndrome; therapeutics; splints; ultrasound therapy.

Level of Evidence: II

Recibido el 15-4-2019. Aceptado luego de la evaluación el 5-5-2020 • Lic. DANIEL HERNANDEZ • danifhernandez@ hotmail.com ID https://orcid.org/0000-0002-0686-338X Cómo citar este artículo: Hernandez D, Terpolilli E, Verdecchia DH, Leiva V, Ventos L, Rodríguez R, et al. Eficacia de la férula nocturna y el ultrasonido para tratar el síndrome del túnel carpiano. Estudio clínico controlado y aleatorizado. Rev Asoc Argent Ortop Traumatol 2020;85(4):357-368. https://doi.org/10.15417/issn. 1852-7434.2020.85.4.982 


\section{INTRODUCCIÓN}

El síndrome del túnel carpiano (STC) o neuropatía de compresión del nervio mediano en la muñeca representa aproximadamente el $90 \%$ de todas las neuropatías por atrapamiento. Se trata de una reconocida causa de discapacidad laboral y se asocia con considerables costos en salud. Su incidencia y prevalencia varían del $0,125 \%$ al $1 \%$ y del $5 \%$ al 16\%, respectivamente, según los criterios utilizados para el diagnóstico. Afecta más a las personas de entre 40 y 60 años, y es más frecuente en las mujeres. ${ }^{1}$

La compresión mecánica del nervio en el túnel carpiano se produce por varios factores, como esfuerzos de presión, sobreuso, prolongada extensión de la muñeca o trabajo manual no habitual. La presión extraneural elevada puede inhibir el flujo sanguíneo intraneural, el transporte axonal y la función neural; y provocar edema endoneural y alteraciones en la mielina. Causa una pérdida lenta de la capacidad de transmitir impulsos nerviosos y lleva eventualmente a la formación de tejido fibroso en el nervio o a cambios en tejidos adyacentes. El STC es idiopático, aunque suele asociarse a cuadros, como diabetes, embarazo, artritis reumatoide, fractura de radio distal, obesidad, diálisis, hipotiroidismo, uso de anticonceptivos orales u otros medicamentos que causen retención de líquidos. Los síntomas iniciales leves e infrecuentes incluyen parestesias, dolor, pérdida de la sensibilidad o debilidad, que suelen empeorar por la noche y, a veces, despiertan al paciente. Si no se tratan, los síntomas suelen tornarse más frecuentes y progresar a entumecimiento, debilidad y atrofia muscular. A causa de los síntomas, los pacientes pierden la capacidad de realizar tareas motoras finas de la mano, como el agarre y la pinza, por lo que disminuye su calidad de vida. El diagnóstico se basa en la evaluación clínica y se confirma con una electromiografía. ${ }^{1-3}$ El tratamiento del STC puede ser quirúrgico o conservador. Se suele ofrecer la cirugía a pacientes con síntomas persistentes, alteración grave de la sensibilidad o debilidad muscular tenar. Existen varias opciones terapéuticas no quirúrgicas, la aplicación de ultrasonido es una de ellas. Esta terapia en un rango de 0,5-2,0 W/ $\mathrm{cm}^{2}$ puede inducir varios efectos biofísicos en los tejidos, como antinflamatorio; mejorar el flujo sanguíneo y la permeabilidad de membrana, estimular la regeneración del nervio y su conducción nerviosa. ${ }^{1,4-7}$ En algunos estudios, otro tratamiento conservador frecuente y eficaz es el uso de férulas. Inmovilizar la muñeca en posición neutra con una férula maximiza el volumen del túnel carpiano y reduce, al mínimo, la presión del nervio mediano, esto alivia el dolor generado por la compresión del nervio, especialmente durante la noche, cuando se coloca la muñeca en flexión. ${ }^{8-12}$

No hay consenso sobre el mejor tratamiento conservador para el STC; asimismo, la evidencia es insuficiente o limitada para justificar el uso del ultrasonido en forma aislada o combinado con otros tratamientos no quirúrgicos, como las férulas, y se necesitan más estudios controlados aleatorizados sobre el tratamiento conservador. ${ }^{7,10}$ Por lo tanto, el objetivo de este estudio fue comparar la eficacia de la inmovilización de la muñeca con una férula de yeso cubital en ángulo neutro durante el descanso nocturno junto con la aplicación de ultrasonido en pacientes con diagnóstico clínico y electromiográfico de STC leve y moderado.

\section{MATERIALES Y MÉTODOS}

Se llevó a cabo un estudio clínico, controlado, aleatorizado con grupo paralelo y con evaluador a ciego, desde octubre de 2007 hasta marzo de 2010. Se incluyó, en forma consecutiva, a pacientes >18 años con diagnóstico de STC sobre la base de los hallazgos clínicos y confirmado por electromiografía, derivados por un traumatólogo para realizar kinesiología en nuestra institución. Los pacientes debían tener síntomas subjetivos (dolor, parestesias o hipoestesias) en el territorio de inervación del nervio mediano y, al menos, una de las siguientes pruebas clínicas de provocación positivas: test de Phalen (flexión de muñeca con adormecimiento o cosquilleo antes de los 60 segundos), signo de Tinel (golpeteos en el nervio mediano a nivel de la muñeca que generan hormigueo o cosquilleo), test de compresión del carpo de Durkan (compresión del túnel carpiano con los pulgares que genera adormecimiento o cosquilleo antes de los 30 segundos). ${ }^{13}$

Se excluyó a pacientes con antecedentes de trastornos metabólicos (como diabetes o hipotiroidismo), artritis reumatoide, infiltración con corticoides, cirugía, diálisis, trauma o fractura de muñeca, férula, que estuvieran recibiendo otro tratamiento para el STC (farmacológico o no farmacológico), embarazadas, o con problemas de comprensión, atrofia tenar severa u otro dolor en la mano que no fuera producido por el STC.

Se eliminó a los pacientes si 1) no concurrían a dos sesiones consecutivas o a tres alternas durante el tratamiento, 2) decidieron abandonar el estudio, 3) recibían cualquier otro tratamiento para este síndrome o fueron diagnosticados de una enfermedad sistémica u otro cuadro en el miembro superior tratado durante el período de estudio, 4) se ausentaron a alguna evaluación durante el tratamiento y 5) usaban la férula después del tratamiento.

El estudio fue aprobado por el Comité de Ética en Investigación de nuestro hospital y todos los pacientes firmaron el consentimiento informado conforme a las normas de ética de la Declaración de Helsinki de 1975. 
Las variables de estudio fueron: dolor, parestesia, la escala PSFS (Patient Specific Functional Scale) y el test de Moberg. El dolor y la parestesia se evaluaron mediante una escala analógica visual de $100 \mathrm{~mm}$ para cada variable por separado, en la que los pacientes podían indicar la intensidad del dolor o la parestesia, en una línea de $10 \mathrm{~cm}$, donde 0 indica "ningún dolor o parestesia" y 10, "el máximo dolor o parestesia que pudiera sentir". ${ }^{14}$

La escala PSFS es un instrumento de autoevaluación que busca identificar el estado funcional y las limitaciones más importantes para el paciente. El evaluador le pide al paciente que identifique hasta cinco actividades importantes que no pueda realizar o con las que tenga alguna dificultad a causa del STC y posteriormente puntúa en una escala numérica de 0 a $10(0=$ imposible de realizar la actividad y $10=$ realiza la actividad como antes del problema). ${ }^{15,16}$ En este estudio, se utilizó una versión traducida de la escala PSFS (Anexo).

\section{ANEXO}

Escala funcional específica del paciente

(versión traducida del Patient Specific Functional Scale)

El profesional debe leer y llenar lo que sigue. Completar al final de la anamnesis y antes del examen físico.

\section{Evaluación inicial:}

Voy a pedirle que identifique hasta cinco actividades importantes que Ud. no puede realizar o que hace con dificultad por su problema de

Hoy, ¿hay alguna actividad que no puede hacer o que hace con dificultad por causa de su problema? (Muestre la escala al paciente y pídale que indique un puntaje para cada actividad.)

\section{Evaluaciones de seguimiento:}

Cuando lo evalué el día (indique la fecha de la evaluación previa), me dijo que tenía dificultad para (lea todas las actividad de la lista).

Hoy, ¿sigue teniendo dificultad para: (lea la lista de actividades y permita al paciente volver a indicar un puntaje en cada una)?

\section{Esquema de puntaje de actividades específicas del paciente (indique el número):}

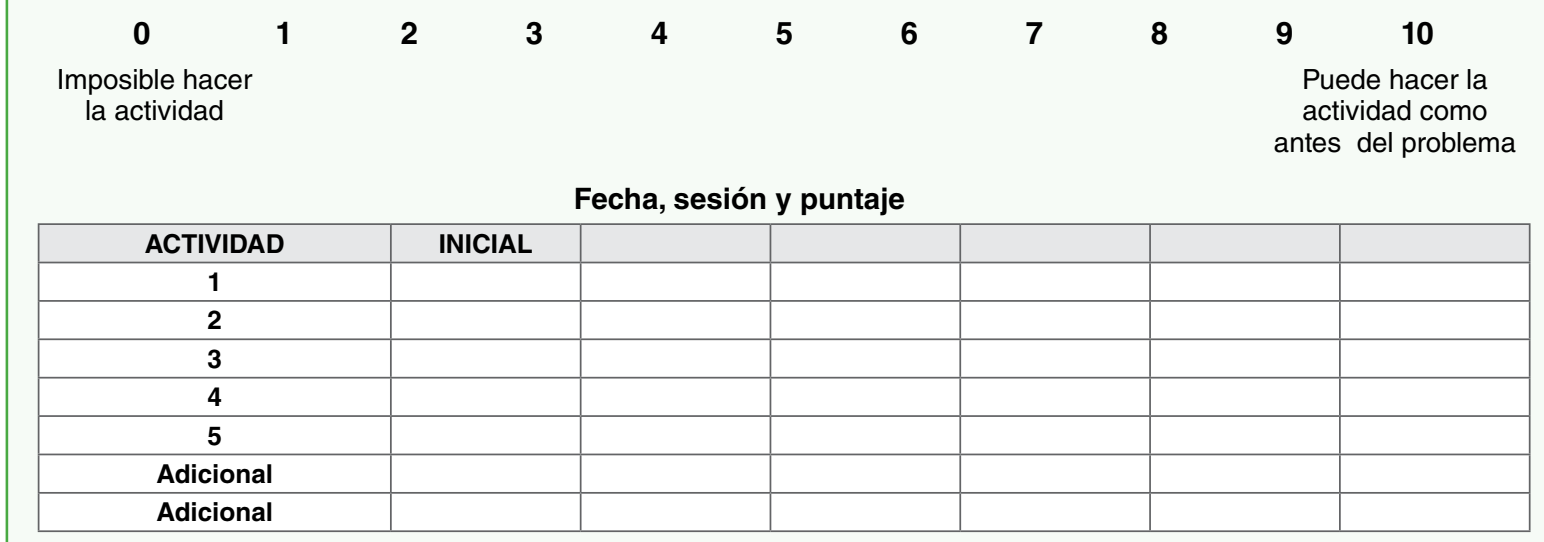

El test de Moberg es una prueba de sensibilidad funcional, en la que se cronometra el tiempo que tarda una persona en tomar con pinza fina 12 objetos y depositarlos en un recipiente. ${ }^{17,18}$ En este estudio, se tomó solo con los ojos abiertos y del lado afectado; los objetos fueron: tornillo mariposa, tornillo, llave, alfiler, tuerca hexagonal grande, tuerca hexagonal chica, tuerca cuadrada chica, moneda grande ( 50 centavos), moneda chica (10 centavos), arandela, alfiler de gancho y clip de papeles. Se le explicó al paciente que debía tomar un objeto a la vez con pinza fina, sin arrastrarlo para agarrarlo, y colocarlos a todos, lo más rápido posible, en el recipiente que estaba ubicado del lado de la mano afectada. A continuación, se cronometró el tiempo que tardaba el paciente en reconocer y nombrar los mismos objetos con los ojos cerrados, y se registró la cantidad de errores.

Se realizó una aleatorización estratificada con bloques permutados aleatorios, el apareamiento de los pacientes fue por sexo y edad ( $\geq 55$ años y $<55$ años), usando cuatro cajas (según apareamiento) con seis sobres opacos cerrados para cada una (tres por grupo). Un kinesiólogo ajeno a la evaluación de los resultados asignó a los pacientes, 
en forma aleatorizada, a dos grupos: el grupo experimental (GE) tratado con ultrasonido y férula nocturna o el grupo de control (GC) que recibía solo ultrasonido. Si el paciente tenía STC bilateral, se trataba primero el lado más sintomático. Al finalizar el tratamiento (a las 6 semanas) y si respetaban los criterios correspondientes para ingresar en el estudio, se incluía el lado no tratado como un nuevo caso de STC.

Un kinesiólogo del grupo evaluador se encargó de examinar a los pacientes al comenzar el tratamiento (T0), a las tres semanas (T1) y al finalizar, a las seis semanas (T2). Luego se realizó un seguimiento al mes (T3), a los tres (T4) y a los seis meses (T5). En la evaluación inicial, además de las variables de estudio, se registraron los datos personales (edad, sexo, lado dominante), las características del dolor, las horas e interrupciones del sueño, la evolución de los síntomas, los datos electromiográficos, las pruebas de provocación, el test de Spurling, de distracción cervical y de abducción del miembro superior; el diagrama de Katz y los antecedentes patológicos. Posteriormente, en cada tiempo de medición, solo se evaluaron las variables del estudio.

Aquellos que analizaban los resultados fueron cegados a la asignación de grupos.

El tratamiento tenía una duración de seis semanas. Tres veces por semana se aplicaba ultrasonido a todos los pacientes, durante 15 min por sesión, sobre el área del túnel carpiano (total 18 sesiones). Se utilizó un equipo de 1 MHz de frecuencia (Sonotherp 990®, Meditea Electromédica S.R.L., Argentina) con una intensidad de 1 W/cm², modalidad pulsante con un transductor de $5 \mathrm{~cm}^{2}$ y un gel a base de agua con $\mathrm{pH}$ neutro como sustancia de acople entre la piel y el cabezal. La aplicación se realizó con el cabezal estático. Los pacientes del GE, además, usaron una férula de yeso confeccionada a medida (con un espesor de 8 vueltas de venda), en ángulo neutro de muñeca, que se extendía por el borde cubital del antebrazo en forma de canal desde la unión del tercio proximal y medio hasta la línea mediopalmar, dejando libre la eminencia tenar y los dedos, cubierta de guata fijada con pegamento en el interior, cinta en los rebordes y sujetada a la muñeca por tres tiras de velcro. Se le indicó al paciente que la usara durante el descanso nocturno, por un período de seis semanas, y que se comunicara con el terapeuta si consideraba que la férula necesitaba un ajuste, el dolor aumentaba al usarla o si tenía algún efecto adverso (Figura 1).

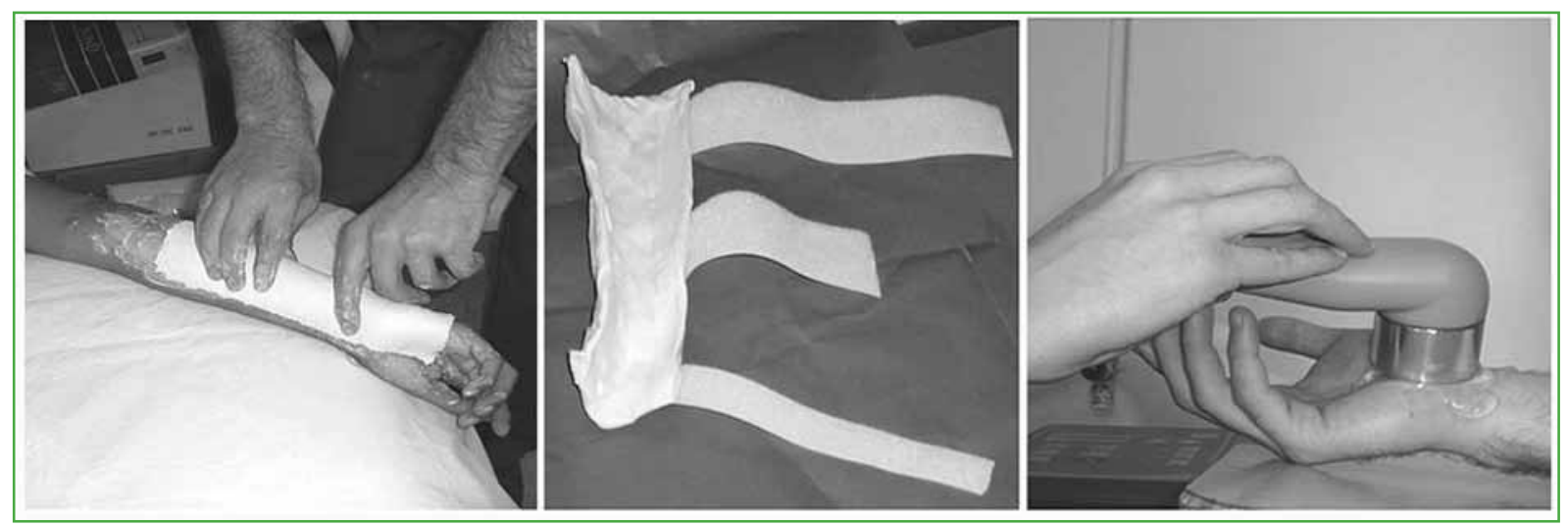

Figura 1. Tratamiento del grupo experimental. Confección de la férula de yeso cubital en ángulo neutro para uso nocturno y aplicación estática de ultrasonido en el túnel carpiano.

Para el análisis estadístico las variables continuas se expresan con su media y desviación estándar (DE), a menos que se indique lo contrario. Las variables categóricas se expresan con su frecuencia y porcentaje. Las características demográficas entre ambos grupos fueron comparadas con el test t de Student o el test U de Mann-Whitney, según correspondiera. Para las variables categóricas se utilizó el test ji al cuadrado o el test exacto de Fisher, según correspondiera. El análisis principal se realizó y se informa de acuerdo con el principio de intención de tratar. En caso de datos faltantes se imputó el último valor registrado de cada paciente. Se llevaron a cabo los correspondientes análisis de sensibilidad con el objetivo de evaluar la robustez de los resultados. El dolor referido, la gravedad de las parestesias, la funcionalidad y el test de Moberg de ambos grupos fueron comparados con un análisis de la covarianza (ANCOVA) ajustando por sexo, edad, dominancia del miembro superior, tiempo de evolución y valores iniciales de cada variable.

Se realizó un modelo mixto de medidas repetidas para el dolor referido, la gravedad de las parestesias, la funcionalidad y el test de Moberg. El modelo incluyó el sexo, la edad, la dominancia del miembro superior, el tiempo 
de evolución y las variables basales como covariables. Los resultados se presentan como diferencias de medias del cambio y sus correspondientes intervalos de confianza del 95\%. Se consideró significativo un valor $\mathrm{p}<0,05$. Para el análisis de los datos se utilizó el programa IBM SPSS Macintosh, versión 23.0 (IBM Corp. Armonk, NY, EE.UU.).

\section{RESULTADOS}

Se incluyó a 65 pacientes, 20 de ellos tenían STC bilateral (16 mujeres y 4 hombres). De 85 casos con STC, 42 fueron asignados, en forma aleatorizada, al GC y 43, al GE. Después de la aleatorización, los pacientes iniciaron la intervención en menos de una semana. Veinte pacientes fueron eliminados, 19 de ellos no completaron tres semanas de tratamiento. De esta manera, el análisis incluyó 32 casos en el GC y 33 en el GE (Figura 2).

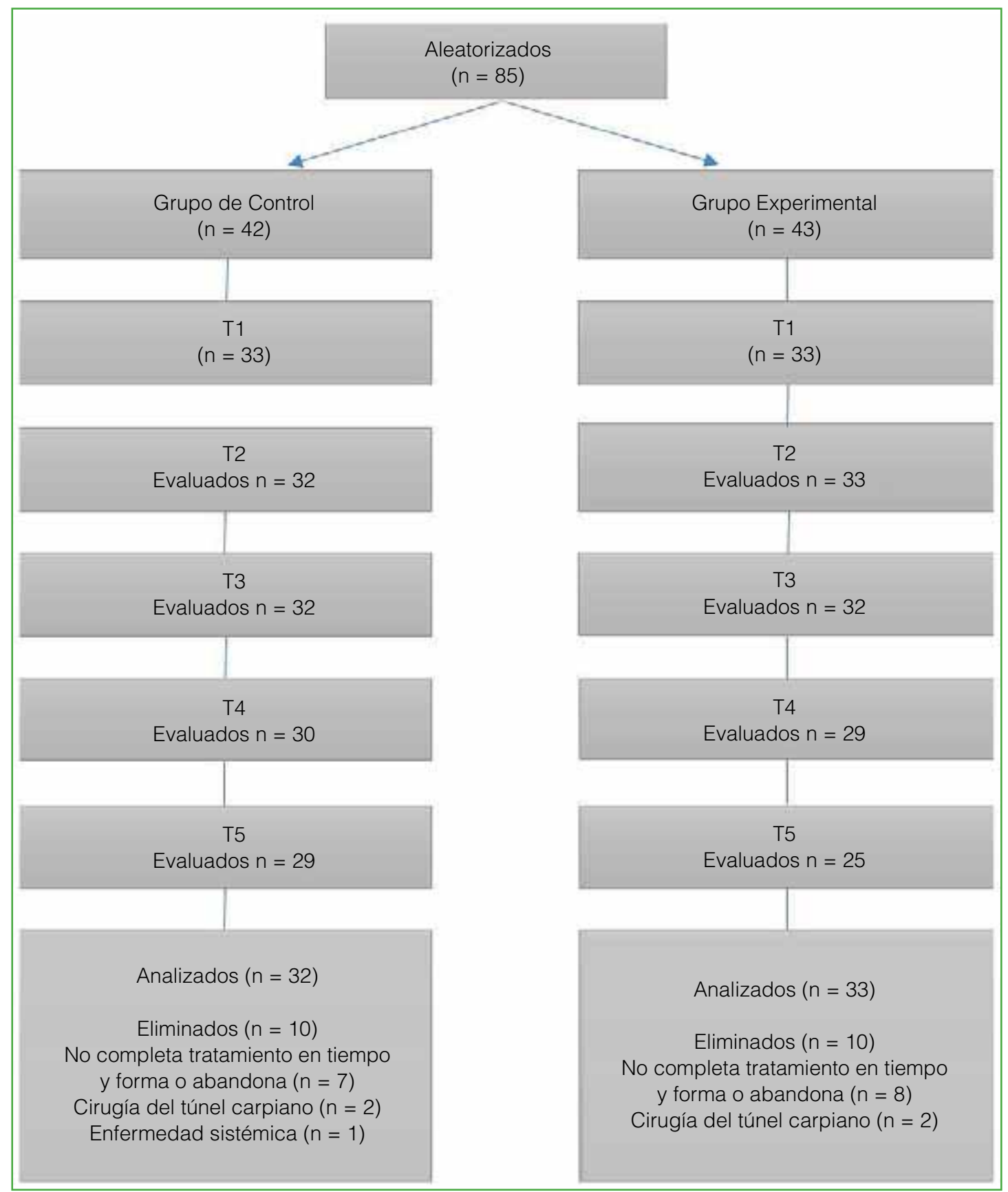

Figura 2. Diagrama de flujo de los pacientes durante el estudio. 
Cincuenta y cuatro $(83 \%)$ asistieron al seguimiento de seis meses tras finalizar el tratamiento. Las características iniciales fueron similares en ambos grupos, excepto por la velocidad de conducción motora en la electromiografía que fue levemente superior en el GE $(53 \mathrm{~m} / \mathrm{s}$ vs. $51 \mathrm{~m} / \mathrm{s} ; \mathrm{p}=0,048)$. El resto de las características iniciales se detalla en la Tabla 1. Las variables dolor y parestesia se muestran en las Tablas 2 y 3, respectivamente. El dolor mejoró en el tiempo, con ambos tratamientos, con diferencias estadísticamente significativas a T2, con diferencia de medias: 1,78 (IC95\% 0,28-3,27, p = 0,009) y en T3 con diferencia de medias: 1,77 (IC95\% 0,46-3,08, p=0,002). Esta diferencia no se mantuvo en T4 y T5.

Tabla 1. Características iniciales de los pacientes analizados

\begin{tabular}{|c|c|c|c|}
\hline Características & $\begin{array}{l}\text { Grupo Experimental } \\
\qquad(\mathbf{n}=\mathbf{3 3})\end{array}$ & $\begin{array}{l}\text { Grupo de Control } \\
\qquad(\mathbf{n}=\mathbf{3 2})\end{array}$ & $\mathbf{p}$ \\
\hline Mujeres & $84,8 \%$ & $75,8 \%$ & 0,35 \\
\hline$\geq 55$ años & $30,3 \%$ & $33,3 \%$ & 0,79 \\
\hline$<55$ años & $69,7 \%$ & $66,7 \%$ & 0,79 \\
\hline $\begin{array}{l}\text { Tiempo de evolución de los síntomas, meses } \\
\text { (mediana, RIC) }\end{array}$ & $12(5.5-30)$ & $12(4.5-42)$ & 0,99 \\
\hline Lado derecho afectado & $63,6 \%$ & $42,4 \%$ & 0,08 \\
\hline Miembro superior derecho dominante & $97 \%$ & $97 \%$ & 0,75 \\
\hline Dolor nocturno & $63,6 \%$ & $66,7 \%$ & 0,8 \\
\hline Dolor en reposo & $39,4 \%$ & $48,5 \%$ & 0,46 \\
\hline Dolor en actividad & $66,7 \%$ & $75,8 \%$ & 0,41 \\
\hline Test de Phalen positivo & $97 \%$ & $84,8 \%$ & 0,098 \\
\hline Test de Tinel positivo & $36,4 \%$ & $45,5 \%$ & 0,45 \\
\hline Test de compresión del carpo Durkan positivo & $90,9 \%$ & $84,8 \%$ & 0,35 \\
\hline Horas de sueño (mediana, RIC) & $7(6-8)$ & $7(5-7.8)$ & 0,69 \\
\hline Interrupción del sueño (mediana, RIC) & $1,5(0-2,5)$ & $1,5(0-3)$ & 0,62 \\
\hline Diagrama de Katz clásico & $12.5 \%$ & $12.1 \%$ & \multirow{3}{*}{0,22} \\
\hline Diagrama de Katz probable & $87,5 \%$ & $78,8 \%$ & \\
\hline Diagrama de Katz posible & $0 \%$ & $9.1 \%$ & \\
\hline
\end{tabular}

RIC = rango intercuartílico 
Tabla 2. Escala analógica visual para dolor

\begin{tabular}{|l|c|c|c|c|c|}
\hline Tiempo & $\begin{array}{c}\text { Grupo } \\
\text { de Control }\end{array}$ & $\begin{array}{c}\text { Grupo } \\
\text { Experimental }\end{array}$ & $\begin{array}{c}\text { Diferencia de } \\
\text { medias (IC95\%) }\end{array}$ & p & $\begin{array}{c}\text { Cambio en el tiempo } \\
\text { de media }(\text { IC95\% }\end{array}$ \\
\hline Inicio
\end{tabular}

${ }^{a}$ Análisis de covarianza ajustado por características basales. ${ }^{b}$ Análisis de un modelo mixto del cambio en el tiempo para las diferencias entre ambos grupos, ajustando por las características basales.

NS = No significativo.

Tabla 3. Escala analógica visual para parestesia

\begin{tabular}{|c|c|c|c|c|c|c|}
\hline Tiempo & $\begin{array}{c}\text { Grupo } \\
\text { de Control }\end{array}$ & $\begin{array}{c}\text { Grupo } \\
\text { Experimental }\end{array}$ & $\begin{array}{c}\text { Diferencia de } \\
\text { medias (IC95\%) }\end{array}$ & p & $\begin{array}{l}\text { Cambio en el } \\
\text { tiempo de media } \\
(\mathrm{IC95} \%)^{\mathbf{b}}\end{array}$ & p \\
\hline Inicio & $6,02(3,07)$ & $6,47(7,18)$ & $-0,41(-3,21 ; 2,38)$ & 0,77 & Referencia & - \\
\hline 3 semanas & $4,22(3,45)$ & $3,77(3,02)$ & $0,47(-1,01 ; 1,95)$ & 0,53 & $2,25(0,25 ; 4,25)$ & 0,016 \\
\hline 6 semanas & $3,64(3,21)$ & $3,39(2,95)$ & $0,32(-1,09 ; 1,73)$ & 0,65 & $2,73(0,53 ; 4,92)$ & 0,005 \\
\hline $\begin{array}{l}1 \text { mes } \\
\text { postratamiento }\end{array}$ & $3,64(3,02)$ & $3,5(3,15)$ & $0(-1,44 ; 1,44)$ & NS & $2,68(0,34 ; 5,02)$ & 0,013 \\
\hline $\begin{array}{l}3 \text { meses } \\
\text { postratamiento }\end{array}$ & $4,14(3,41)$ & $3,3(3,19)$ & $1,04(-0,5 ; 2,6)$ & 0,18 & $2,52(0,16 ; 4,89)$ & 0,027 \\
\hline $\begin{array}{l}6 \text { meses } \\
\text { postratamiento }\end{array}$ & $4,66(3,72)$ & $3,61(3,21)$ & $1,16(-0,5 ; 2,82)$ & 0,17 & $2,12(-0,21 ; 4,44)$ & 0,11 \\
\hline
\end{tabular}

${ }^{a}$ Análisis de covarianza ajustado por características basales. ${ }^{b}$ Análisis de un modelo mixto del cambio en el tiempo para las diferencias entre ambos grupos, ajustando por las características basales.

NS = No significativo. 
No se observaron diferencias estadísticamente significativas entre los grupos en ninguna de las variables estudiadas, a excepción del dolor a favor del GE en T1 con diferencia de medias: 1,64 (IC95\% 0,38-2,91, p = 0,012) (Figura 3A).

La media de parestesias en T0 fue de 6,02 (DE 3,07) para el GC y de 6,47 (DE 7,18) para el GE (p = 0,77). Los síntomas de parestesias mejoraron con el tiempo en ambos grupos, con diferencias estadísticamente significativas en todas las evaluaciones, pero no en T5. No se observaron diferencias estadísticamente significativas entre ambos grupos (Figura 3B).

La media del tiempo para completar el test de Moberg en T0 fue de 25,88 segundos (DE 13,94) para el GC y de 25,39 segundos (DE 10,23) para el GE ( $\mathrm{p}=0,98)$. A lo largo del tiempo, el desempeño mejoró en ambos grupos al compararlo con la prueba inicial, pero solo a partir de T2, se observaron diferencias estadísticamente significativas (Figura 3C). No hubo diferencias estadísticamente significativas entre ambos grupos en ninguna de las evaluaciones respecto al tiempo para realizar el test, el tiempo de reconocimiento de objetos o la cantidad de errores.

En la escala PSFS, las tres actividades elegidas con más frecuencia fueron: levantar elementos pesados (38 casos), escurrir un trapo (32 casos) y lavar ropa a mano (18 casos). Con el tiempo, los resultados mejoraron en ambos grupos al compararlos con los puntajes iniciales, con diferencias estadísticamente significativas, pero sin diferencia significativa entre grupos (Figura 3D). Solo hubo diferencias significativas para la Actividad 1 en T5 $(\mathrm{GE}=8$ y $\mathrm{GC}=5, \mathrm{p}=0,038)$. No se informaron efectos adversos ni complicaciones.

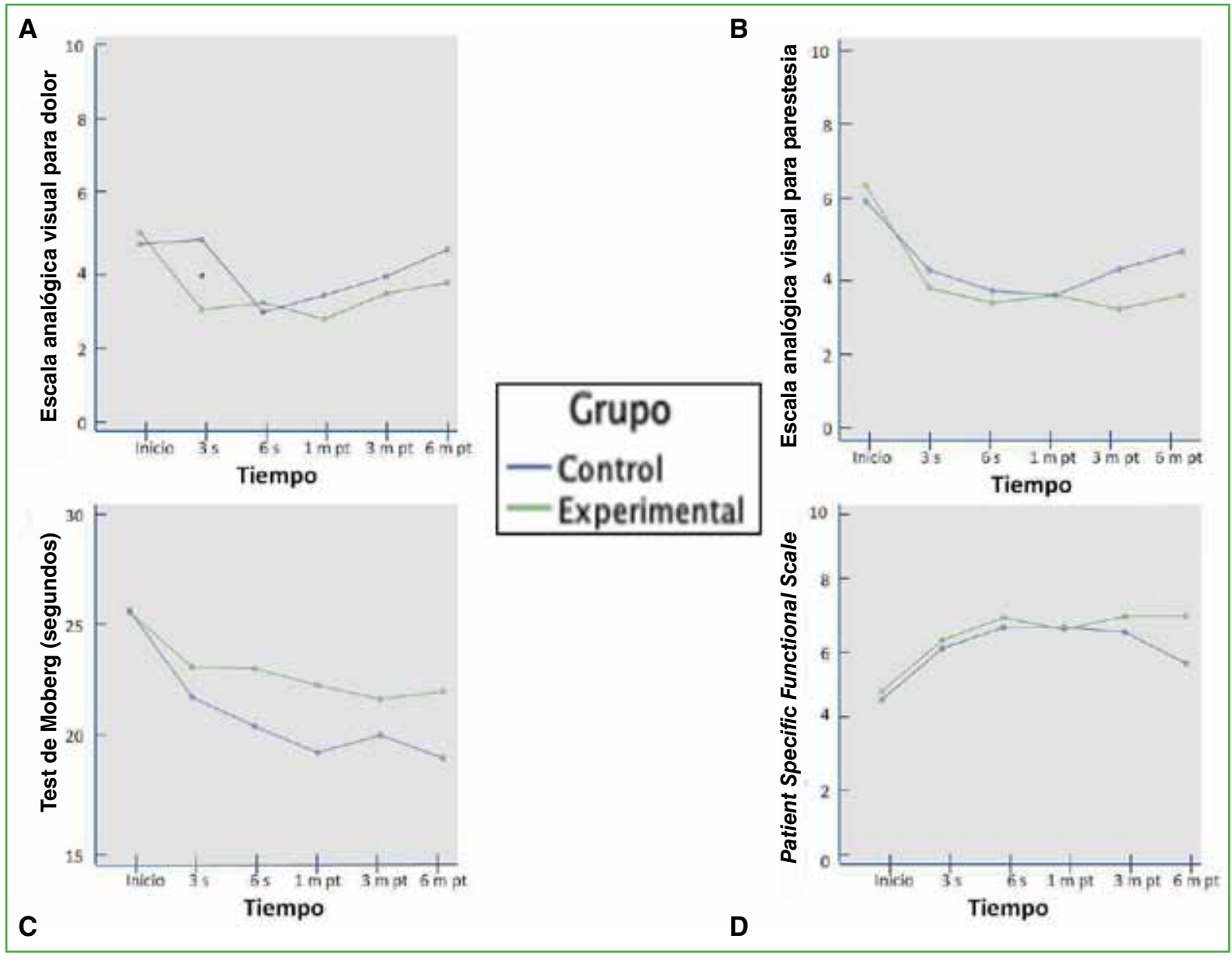

Figura 3. Comportamiento de las variables en el tiempo. Dolor, parestesia, test de Moberg y Patient Specific Functional Scale. ${ }^{*}$ Significativo; $\mathrm{s}=$ semanas, $\mathrm{m} \mathrm{pt}=$ mes posterior al tratamiento. 


\section{DISCUSIÓN}

Los resultados sugieren que la inmovilización nocturna de la muñeca con una férula de yeso cubital en ángulo neutro y la aplicación de ultrasonido puede ser un tratamiento beneficioso para pacientes con STC.

El STC afecta con más frecuencia a personas $>30$ años, particularmente a las mujeres, ${ }^{19}$ lo que concuerda con nuestra muestra analizada, ya que el $80 \%$ eran mujeres y nadie tenía menos de 30 años.

No hay consenso sobre el tratamiento inicial del STC, puede ser quirúrgico o conservador. El tratamiento conservador de elección es controvertido, porque se han comunicado varios tratamientos ineficaces, insuficientes o con resultados contradictorios. ${ }^{1,7,11,19,20}$ Sin embargo, hay mayor interés por el tratamiento conservador debido al riesgo y la alta tasa de recurrencia de los procedimientos quirúrgicos, la evolución natural de la enfermedad que, a veces, se cura espontáneamente, los bajos costos y las raras complicaciones que este tipo de tratamiento provoca. Por todo esto, se recomienda un abordaje conservador al comenzar a tratar el STC, especialmente para los casos leves o moderados. ${ }^{21,22}$

Como ninguna intervención puede considerarse el patrón de referencia en el tratamiento conservador del STC, a los pacientes de los GC y GE se les aplicó ultrasonido, un equipo de fisioterapia accesible y ampliamente utilizado en la práctica kinésica. Se ha demostrado que el ultrasonido es más eficaz que no administrar tratamiento y que otras terapias. Si bien no hay consenso sobre cuál es la dosis óptima para tratar el STC; ${ }^{6,7,23-25}$ en este estudio, se implementó una modalidad terapéutica de ultrasonido de $1 \mathrm{MHz}$, pulsátil de $1 \mathrm{~W} / \mathrm{cm}^{2}$, durante $15 \mathrm{~min}$, tres veces por semana, por seis semanas (18 sesiones), porque también se demostró que es un tratamiento eficaz que logra resultados satisfactorios a corto y mediano plazo en pacientes con STC idiopático leve o moderado. ${ }^{6,725} \mathrm{En}$ la población de nuestro estudio, la aplicación de ultrasonido con el uso de una férula o sin ella produjo cambios significativos al finalizar el tratamiento. Estos hallazgos coinciden con los de Ebenbichler y cols., quienes hallaron diferencias significativas con respecto al grupo placebo luego de 20 sesiones de ultrasonido pulsátil. ${ }^{6}$

El uso de una férula es otro tratamiento frecuente y no hay evidencia de eficacia entre su uso permanente o nocturno. ${ }^{23}$ En este estudio, se inmovilizó la muñeca con una férula de yeso cubital confeccionada a medida, en ángulo neutro, para que los pacientes la usaran por la noche, ya que el retorno venoso disminuye y la presión en el túnel del carpo se incrementa durante el sueño, debido a la menor actividad o la posición anómala; este puede ser el motivo de la frecuencia de los síntomas nocturnos en estos pacientes. Se demostró que la posición neutra es la que genera menor presión en el túnel carpiano y el nervio mediano. Se ha informado que se prefieren las férulas en forma de canal colocadas en el borde cubital a las férulas volares hasta la punta de los dedos, porque dejan los dedos libres y permiten cierto uso de la mano. ${ }^{8-12,21}$ La férula se confeccionó con yeso debido a su bajo costo y porque era el único material disponible en una institución pública. La férula nocturna en el GE produjo cambios significativos al final del tratamiento, lo que coincide con otras investigaciones. ${ }^{9-11}$ Sin embargo, en nuestro estudio, no es posible atribuir los beneficios exclusivamente al uso de la férula, porque el GE recibió además el tratamiento con ultrasonido.

Como los síntomas del STC suelen empeorar si no se trata el cuadro y tanto el ultrasonido como la férula son más eficaces que no administrar tratamiento, ${ }^{2,23}$ no incluimos un grupo placebo, ya que no consideramos ético dejar sin tratamiento a un paciente cuando existe un tratamiento sustentado por la evidencia científica.

Muy pocos estudios evalúan los resultados luego de los tres meses de tratamiento con ultrasonido o férula en el STC. En nuestro estudio, se informan resultados hasta seis meses después del tratamiento y no se han reportado efectos adversos o complicaciones por el uso del ultrasonido o de la férula, dato que concuerda con los de estudios publicados.,10

Nuestros resultados coinciden con los de algunos estudios en los que el tratamiento con ultrasonido o férula mejora los síntomas de pacientes con STC. ${ }^{6,9,11,19,21,25}$ En la comparación intragrupos, se hallaron diferencias significativas entre T0 y T3, T0 y T4 en todas las variables, excepto en el tiempo de tomar-poner del test de Moberg.

No se observaron diferencias estadísticamente significativas entre los grupos, en ninguna de las variables estudiadas, a excepción del dolor a favor del GE en T1, con diferencia de medias: 1,64 (IC95\% 0,38-2,91, p = 0,012). Sin embargo, la diferencia fue pequeña y no alcanzó la mínima diferencia clínica significativa. La intensidad del dolor y la depresión se asocian con la función de la mano. La depresión es un factor psicológico asociado con la intensidad del dolor, que no se evaluó en este estudio, y que puede influir en los resultados.

Los resultados del test de Moberg no pueden compararse con los de otros estudios, ya que, si bien es considerada una prueba simple y rápida para la evaluación funcional de la mano, no se había utilizado en pacientes con STC. En este estudio, se tomó solo con los ojos abiertos del lado por tratar, aunque con el tiempo, el desempeño mejoró en ambos grupos al compararlo con la evaluación inicial. Solo a partir de T2, se observaron diferencias estadísticamente significativas a favor del GC, pero no hubo diferencias estadísticamente significativas entre los grupos en ninguna de las evaluaciones (tiempo de reconocimiento de objetos y cantidad de errores). 
En la escala PSFS, no se hallaron diferencias significativas en ninguno de los tiempos de medición entre los grupos, excepto en T5 donde en la Actividad 1 se encontró una diferencia significativa a favor del GE.

Se recomienda usar la férula a pacientes con STC dentro de los tres meses de la aparición de los síntomas, ya que es más eficaz. ${ }^{9}$ En nuestro estudio, no se tuvo en cuenta el tiempo de evolución de los síntomas en el momento de la aleatorización. Otras limitaciones fueron el tamaño pequeño de la muestra, la tasa elevada de abandono del tratamiento, no tener en cuenta las variables psicosociales ni la gravedad de los síntomas, que pueden influir en los resultados; el empleo de la escala PSFS que no estaba adaptada ni validada para su uso en la Argentina, en ese momento, y no evaluar la respuesta terapéutica en relación con el alivio de los síntomas nocturnos, demostrada en otros estudios. ${ }^{7,11}$ Se eliminó al 23,5\% de los casos incluidos, las razones más comunes fueron: no cumplir el tratamiento en tiempo y forma o abandonarlo (

Este estudio se llevó a cabo en pacientes con síntomas leves o moderados de un Servicio de Kinesiología. Se debe ser cuidadoso al generalizar los resultados a pacientes con síntomas más graves. Al trabajar en un hospital público que brinda atención a pacientes que, a veces, residen en áreas lejanas, el gasto y el tiempo de traslado, la duración del tratamiento, la necesidad de volver a trabajar o a las responsabilidades personales lo antes posible, dificultan realizar un seguimiento largo en nuestra práctica clínica y esto podría, al menos en parte, explicar la alta tasa de deserción.

\section{CONCLUSIONES}

La eficacia de la inmovilización de la muñeca con una férula cubital en ángulo neutro durante la noche y la aplicación conjunta de ultrasonido no son superiores al tratamiento solo con ultrasonido, en pacientes con STC leve y moderado al mes y a los tres meses de seguimiento. Se necesitan más estudios de buena calidad científica para determinar la eficacia del uso de una férula y el ultrasonido en el tratamiento del STC, así como de otros tratamientos conservadores.

\section{Agradecimientos}

A los kinesiólogos y al Servicio de Ortopedia y Traumatología de nuestro hospital por el apoyo brindado.

Conflicto de intereses: Los autores no declaran conflictos de intereses.

ORCID de E. Terpolilli: https://orcid.org/0000-0003-2718-8851 ORCID de D. H. Verdecchia: https://orcid.org/0000-0003-1610-4923 ORCID de V. Leiva: https://orcid.org/0000-0001-8158-157X ORCID de L. Ventos: https://orcid.org/0000-0002-7953-9345 ORCID de R. Rodríguez: https://orcid.org/0000-0002-3809-8715 ORCID de A. Gamboa: https://orcid.org/0000-0002-3623-2052 ORCID de M. B. Spath: https://orcid.org/0000-0003-2166-8504 ORCID de J. Steimberg: https://orcid.org/0000-0002-7696-0759 ORCID de M. Sanz: https://orcid.org/0000-0002-1090-4842
ORCID de E. Williams: https://orcid.org/0000-0003-3058-6164 ORCID de S. Mohr: https://orcid.org/0000-0003-3058-6164 ORCID de P. Colesnik: https://orcid.org/0000-0001-5269-0822 ORCID de T. L. Chen: https://orcid.org/0000-0001-8103-7988 ORCID de S. Dell'Era: https://orcid.org/0000-0001-9186-6229 ORCID de R. Díaz: https://orcid.org/0000-0001-9323-6503 ORCID de M. Dimaro: https://orcid.org/0000-0003-3860-6254 ORCID de B. Baldo: https://orcid.org/0000-0003-2633-6349 ORCID de M. Accoce: https://orcid.org/0000-0003-3860-6254 


\section{BIBLIOGRAFÍA}

1. Aroori S, Spence RA. Carpal tunnel syndrome. Ulster Med J 2008;77(1):6-17. PMID: 18269111

2. Burke FD, Ellis J, McKenna H, Bradley MJ. Primary care management of carpal tunnel syndrome. Postgrad Med J 2003;79(934):433-7. https://doi.org/10.1136/pmj.79.934.433

3. Jablecki CK, Andary MT, Floeter MK, Miller RG, Quartly CA, Vennix MJ, et al. Practice parameter: Electrodiagnostic studies in carpal tunnel syndrome. Report of the American Association of Electrodiagnostic Medicine, American Academy of Neurology, and the American Academy of Physical Medicine and Rehabilitation. Neurology 2002;58(11):1589-92. https://doi.org/10.1212/wnl.58.11.1589

4. Currier DP, Greathouse D, Swift T. Sensory nerve conduction: effect of ultrasound. Arch Phys Med Rehabil 1978;59(4):181-5. PMID: 646607

5. Kramer JF. Effect of therapeutic ultrasound intensity on subcutaneous tissue temperature and ulnar nerve conduction velocity. Am J Phys Med 1985;64(1):1-9. PMID: 3970155

6. Ebenbichler GR, Resch KL, Nicolakis P, Wiesinger GF, Uhl F, Ghanem AH, et al. Ultrasound treatment for treating the carpal tunnel syndrome: randomised "sham" controlled trial. BMJ 1998;316(7133):731-5. https://doi.org/10.1136/bmj.316.7133.731

7. Page MJ, O’Connor D, Pitt V, Massy-Westropp N. Therapeutic ultrasound for carpal tunnel syndrome. Cochrane Database Syst Rev 2013;2013( 3):CD009601. https://doi.org/10.1002/14651858.CD009601.pub2

8. Werner CO, Elmqvist D, Ohlin P. Pressure and nerve lesion in the carpal tunnel. Acta Orthop Scand 1983;54(2):312-6. https://doi.org/10.3109/17453678308996576

9. Kruger VL, Kraft GH, Deitz JC, Ameis A, Polissar L. Carpal tunnel syndrome: objective measures and splint use. Arch Phys Med Rehabil 1991;72(7):517-20. PMID: 2059127

10. Page MJ, Massy-Westropp N, O’Connor D, Pitt V. Splinting for carpal tunnel syndrome. Cochrane Database Syst Rev 2012;2012(7):CD010003. https://doi.org/10.1002/14651858.CD010003

11. Halac G, Demir S, Yucel H, Niftaliyev E, Kocaman G, Duruyen H, et al. Splinting is effective for night-only symptomatic carpal tunnel syndrome patients. J Phys Ther Sci 2015;27(4):993-6. https://doi.org/10.1589/jpts.27.993

12. Armenteros Pedrero J, Justo García G, Rey Pita ML, Gallego González S, Buján de Gonzalo A, Tobío Iglesias A. Tratamiento conservador del síndrome del túnel carpiano mediante inmovilización con férulas. Rehabilitación (Madr) 2000; 34(4): 313-9.

https://www.elsevier.es/es-revista-rehabilitacion-120-articulo-tratamiento-conservador-del-sindrome-del-10017830

13. Massy-Westropp N, Grimmer K, Bain G. A systematic review of the clinical diagnostic tests for carpal tunnel syndrome. J Hand Surg Am 2000;25(1):120-7. https://doi.org/10.1053/jhsu.2000.jhsu025a0120

14. Gallagher EJ, Liebman M, Bijur PE. Prospective validation of clinically important changes in pain severity measured on a visual analog scale. Ann Emerg Med 2001;38(6):633-8. https://doi.org/10.1067/mem.2001.118863

15. Stratford P, Gill C, Westaway M, Binkley J. Assessing disability and change on individual patients: a report of a patient specific measure. Physiother Can 1995;47(4):258-63. https://doi.org/10.3138/ptc.47.4.258

16. Hefford C, Abbott JH, Arnold R, Baxter GD. The patient-specific functional scale: validity, reliability, and responsiveness in patients with upper extremity musculoskeletal problems. J Orthop Sports Phys Ther 2012;42(2):56-65. https://doi.org/10.2519/jospt.2012.3953

17. Ng CL, Ho DD, Chow SP. The Moberg pickup test: results of testing with a standard protocol. $J$ Hand Ther 1999;12(4):309-12. https://doi.org/10.1016/s0894-1130(99)80069-6

18. Stamm TA, Ploner A, Machold KP, Smolen J. Moberg picking-up test in patients with inflammatory joint diseases: a survey of suitability in comparison with button test and measures of disease activity. Arthritis Rheum 2003;49(5):626-32. https://doi.org/10.1002/art.11378

19. Baysal O, Altay Z, Ozcan C, Ertem K, Yologlu S, Kayhan A. Comparison of three conservative treatment protocols in carpal tunnel syndrome. Int J Clin Pract 2006;60(7):820-8. https://doi.org/10.1111/j.1742-1241.2006.00867.x

20. Armagan O, Bakilan F, Ozgen M, Mehmetoglu O, Oner S. Effects of placebo-controlled continuous and pulsed ultrasound treatments on carpal tunnel syndrome: a randomized trial. Clinics (Sao Paulo) 2014;69(8):524-8. https://doi.org/10.6061/clinics/2014(08)04

21. Dincer U, Cakar E, Kiralp MZ, Kilac H, Dursun H. The effectiveness of conservative treatments of carpal tunnel syndrome: splinting, ultrasound, and low-level laser therapies. Photomed Laser Surg 2009;27(1):119-25. https://doi.org/10.1089/pho.2008.2211 
22. Fernández-de-Las-Peñas C, Ortega-Santiago R, Díaz HF, Salom-Moreno J, Cleland JA, Pareja JA, et al. Costeffectiveness evaluation of manual physical therapy versus surgery for carpal tunnel syndrome: evidence from a randomized clinical trial. J Orthop Sports Phys Ther 2019;49(2):55-63. https://doi.org/10.2519/jospt.2019.8483

23. Huisstede BM, Hoogvliet P, Randsdorp MS, Glerum S, van Middelkoop M, Koes BW. Carpal tunnel syndrome. Part I: effectiveness of nonsurgical treatments—a systematic review. Arch Phys Med Rehabil 2010;91(7):981-1004. https://doi.org/10.1016/j.apmr.2010.03.022

24. Huisstede BM, Hoogvliet P, Franke TP, Randsdorp MS, Koes BW. Carpal tunnel syndrome: effectiveness of physical therapy and electrophysical modalities. An updated systematic review of randomized controlled trials. Arch Phys Med Rehabil 2018;99(8):1623-34.e23. https://doi.org/10.1016/j.apmr.2017.08.482

25. Bakhtiary AH, Rashidy-Pour A. Ultrasound and laser therapy in the treatment of carpal tunnel syndrome. Aust $J$ Physiother 2004;50(3):147-51. https://doi.org/10.1016/s0004-9514(14)60152-5 